\title{
Growth of deformation twins in room-temperature rolled nanocrystalline nickel
}

\author{
X. Y. Zhang, ${ }^{1,2, a)}$ X. L. Wu, ${ }^{3, b)}$ and A. W. Zhu ${ }^{2}$ \\ ${ }^{1}$ School of Materials Science and Engineering, Chongqing University, Chongqing 400030, \\ People's Republic of China \\ ${ }^{2}$ Physical Science and Engineering Technology School, Guangxi University, Nanning 530004, \\ People's Republic of China \\ ${ }^{3}$ State Key Laboratory of Nonlinear Mechanics, Institute of Mechanics, Chinese Academy of Sciences, \\ Beijing 100190, People's Republic of China
}

(Received 6 January 2009; accepted 3 March 2009; published online 24 March 2009)

\begin{abstract}
Deformation twinning has been observed in room-temperature rolled nanocrystalline Ni. The growth of the deformation twins via the emission of partial dislocations from a grain boundary has been examined in detail. Partial dislocations on neighboring slip planes may migrate for different distances and then remain in the grain interior, leading to the formation of a steplike twin boundary (TB). With continued twin growth, the TBs become gradually distorted and lose their coherent character due to accumulated high stresses. Moreover, we propose that microtwins may form near such TBs due to the emission of partial dislocations from the TB. (C) 2009 American Institute of Physics. [DOI: 10.1063/1.3104858]
\end{abstract}

Twins have been observed in a number of deformed nanocrystalline (nc) metals, including $\mathrm{Cu},{ }^{1} \mathrm{Ta},{ }^{2} \mathrm{Al},{ }^{3,4} \mathrm{Pd},{ }^{5}$ and $\mathrm{Ni}^{6-10}$ even though they are seldom found in the deformation of similar coarse-grained metals. Deformation twinning therefore plays an important role in the deformation of nc materials, and it is important to find out how twins form and grow during deformation. Molecular dynamics (MD) simulations ${ }^{11,12}$ have suggested that grain boundary (GB) emission of partial dislocations can result in deformation twinning in $\mathrm{nc} \mathrm{Cu}$ and $\mathrm{nc} \mathrm{Al}$, as confirmed by some experimental results. ${ }^{1,3,4}$ MD simulations also show that for the deformation of nc Ni (20-50 nm), GB emission of partial dislocations does not result in twinning. ${ }^{11}$ However, deformation twinning has been observed in deformed nc Ni rolled to different strains at low temperature (in liquid nitrogen). ${ }^{6,7,9}$ In this letter, we report that deformation twinning can also take place during room-temperature cold-rolling of nc Ni. The growth of twins by the emission of partial dislocations from GBs is discussed in detail for the cases of three different types of twin boundaries (TBs).

Fully dense, electrodeposited nc Ni sheets were procured from Goodfellow Inc. The as-received sheets were $200 \mu \mathrm{m}$ thick and of $99.8 \%$ purity. The average grain size was about $20 \mathrm{~nm}$. Samples of $\mathrm{nc} \mathrm{Ni}$ of $10 \times 20 \mathrm{~mm}^{2}$ in size were rolled at room temperature to various von Mises equivalent strains $\varepsilon_{\mathrm{VM}}$ calculated as $\varepsilon_{\mathrm{VM}}=|2 / \sqrt{3} \ln (1+\delta)|$, where $\delta$ is the rolling reduction. The equivalent strain rate was approximately $10^{-2} \mathrm{~s}^{-1}$. The microstructures were examined using $\mathrm{x}$-ray diffraction (XRD), transmission electron microscopy (TEM), and high-resolution TEM (HRTEM) (JEM 2010F operated at $200 \mathrm{kV}$ ). The TEM specimens were prepared by twin-jet polishing using a nitric acid-methanol solution $(20 \%$ of $\mathrm{HNO}_{3}$ by volume) at $-30{ }^{\circ} \mathrm{C}$.

The HRTEM experimental results show that the presence of a lot of deformation twins in the cold-rolled samples.

\footnotetext{
a) Electronic mail: kehen888@163.com.

${ }^{b)}$ Electronic mail: xlwu@imech.ac.cn.
}

This result agrees with previously reported results for deformed nc Ni. ${ }^{6-9}$ However, our samples were deformed at room temperature, whereas the samples analyzed in Refs. 6-9 were deformed at low temperature (in liquid nitrogen). Figure 1 shows an example image of a twin (for a sample rolled to $\varepsilon_{\mathrm{VM}}=0.45$ ). The low magnification illustration in the upper left corner of Fig. 1 indicates the twin position. Three grains, A, B, and C, can be seen and the twin is enlarged from the white box in grain A. The twin relationship at TB1 and TB2 is confirmed by the fast Fourier transformation of the lattice image shown in the lower left corner. From Fig. 1 it can be seen that the TB is not straight, as shown by broken line between TB1 and TB2. This kind of TB can be

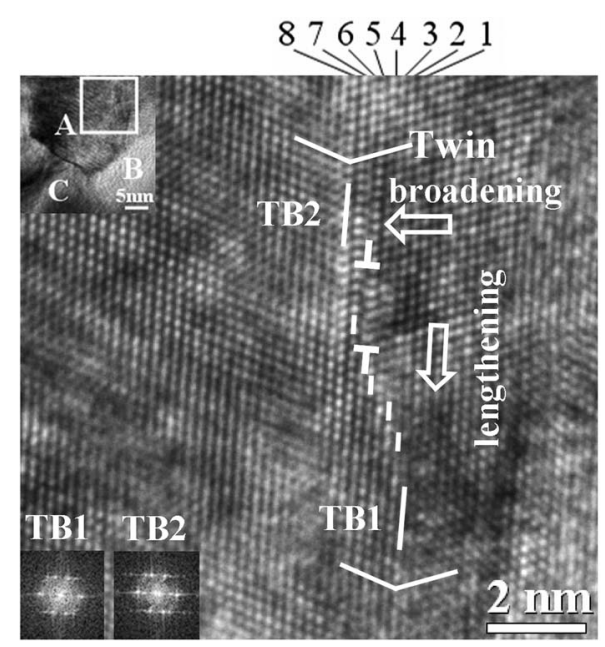

FIG. 1. Twin grows in deformed nc Ni. The win is enlarged from the white box in the upper left corner, in which there are three grains, A, B, and C. The fast Fourier transformation for twin relationship at TB1 and TB2 is shown in the lower right corner. The broken line shows the steplike TB between TB1 and TB2. Note the atomic plane at TB1 is counted as the first plane, with the second to the eighth plane counted in order, the plane at TB2 is the eighth plane. There is a dislocation loop formed by Shockley dislocation marked by $\mathrm{T}$ in the sixth plane. The two arrows indicate twin growing direction in lengthening and broadening. 
regarded as a stepped boundary. It can also be seen that the twin on the right side of TB1 has reached the GB between grains $\mathrm{A}$ and $\mathrm{B}$. However, the twin between TB1 and TB2 remains in the grain interior. In order to explain how the twin grows, we can number the atomic plane of TB1 as the first plane (number 1), with the second plane (number 2) on its left. Counting the atomic planes in order, the atomic plane of TB2 is the eighth plane (number 8), as shown in Fig. 1. We propose that partial dislocations are emitted plane by plane in sequence from the upper GB of grain A (not shown in the figure) and glide downward to the grain interior, leading to the twin broadening and lengthening. Because they are emitted earlier, the partial dislocations of the first atomic plane and the planes to its right have reached the A/B GB. However, the partial dislocations on the second to eighth plane remain in the grain. It is because of this, upon unloading the force to drive them forward, that it disappears and they remain in the grain. Due to the pinning effect of the dislocation loop formed by a Shockley dislocation marked by "T" in Fig. 1 , these partial dislocations cannot retreat to the original GB. It is conceivable that with continued loading, the partial dislocations continue forward the A/B GB. At the same time, partial dislocations on each atomic plane in the left of the eighth plane will be emitted, leading to extension and growth of the twin, as shown by arrows in Fig. 1. Therefore, the step seen in the TB results from the interrupted process of twin growth by a process of the partial dislocation glide.

Steplike TBs, as seen in Fig. 1, have also been reported in literature ${ }^{1}$ (e.g., the a-a TB in Fig. 3 of Ref. 1). In that case the twin was in grain interior, and had not formed either a new grain or two TBs of different direction. ${ }^{13}$ Therefore, it is expected that these twins, similar to the twin shown in Fig. 1, are all the result of the GB emission of partial dislocations. Some partial dislocations remain in the grain interior and induce a stepped TB structure, as discussed above.

We also observed some straight twins with exact lattice coherence TB [e.g., Fig. 2(a) and in the lower left white box of Fig. 2(b)]. Additionally, some other twins were also found to have a TB that cannot be described by one atomic plane, as shown in Figs. 2(b) and 2(c). From Fig. 2 it can be seen that these TBs contain several atomic planes [upper right white box in Figs. 2(b) and 2(c)]. In general, microtwins can be found in these TB regions, implying that the migration of partial dislocations takes place. Furthermore, dislocations marked by $\mathrm{T}$ and dislocation dipoles marked by "dashed circle" in Fig. 2(c) can be observed in these TB regions.

The presence of microtwins inside twins have been reported previously ${ }^{9}$ (Fig. 8 of Ref. 9), and was attributed to the GB emission of partial dislocations. From Figs. 2(b) and 2(c) it can be seen that the stacking faults (SFs) in the TB region are not continuous but interrupted. Additionally there are several dislocations ahead of and behind the microtwins. It is therefore possible that the microtwins are generated from the TB and not the GB. We assume here that for GB emission of partial dislocations, the twin size is limited and cannot grow across the whole grain. In the latter stages of twin growth, a high stress will exist in the twin tip. This stress will hinder partial dislocations emitted by GB (Ref. 14) and interact with the TB, leading to TB distortion. ${ }^{15}$ Such a highly heterogeneous strained region can result in the easy nucleation of dislocations, i.e., the emission of dislocations from a TB. ${ }^{15}$ Interaction of dislocations can produce a Lomer-Cottrell lock. ${ }^{13}$ Subsequently, the Lomer-Cottrell

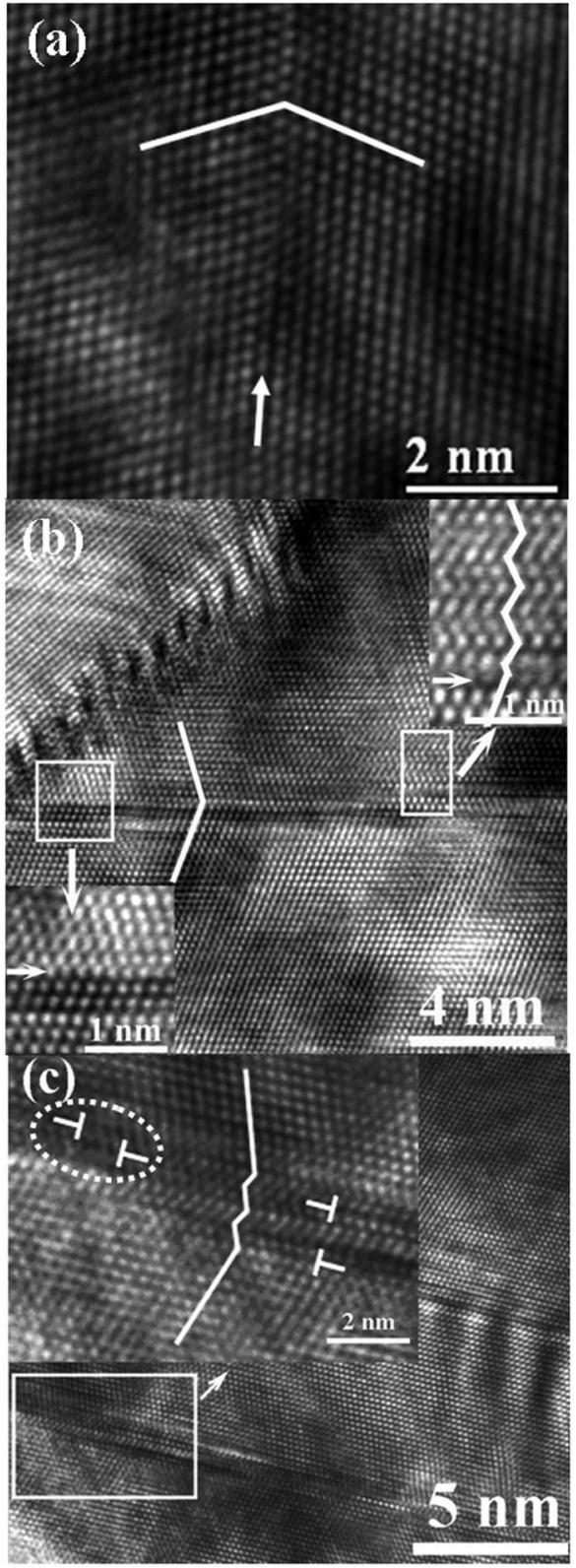

FIG. 2. Twins in deformed nc Ni. (a) Twin with a straight TB. (b) Straight TB (lower left white box) and TB containing several atomic planes and microtwins (upper right white box). (c) Perfect dislocations marked by $\mathrm{T}$ and dislocation dipole marked by dashed circle near TB region.

lock can emit partial dislocations to produce local microtwins and SFs. Thus a discontinuous Lomer-Cottrell lock generated near a TB can lead to the formation of discontinuous and local SFs and microtwins. As a result, the originally straight TBs lose their lattice coherence and become "messy." If plastic strain increases continually, the TB will evolve into a distorted GB with higher energy.,

In summary, in this letter, deformation twinning has been observed in room-temperature cold-rolled nc Ni. Growth of twins by the emission of partial dislocations from GB has been illustrated in detail. If some partial dislocations remain in the grain interior and do not reach the opposite GB, a stepped TB is formed. With increasing twin size, TBs become gradually disordered and distorted by high stresses accumulated at the twin tip. TBs can act as a blockage of partial dislocation glide. The formation of microtwins can be attributed to the emission of dislocations from TB. 
This work was supported by NSFC Grant Nos. 50890174, 50571110, 10721202, and 50461001.

${ }^{1}$ X. Z. Liao, F. Zhou, E. J. Lavernia, D. W. He, and Y. T. Zhu, Appl. Phys. Lett. 83, 5062 (2003).

${ }^{2}$ Y. M. Wang, A. M. Hodge, J. Biener, A. V. Hamza, D. E. Barnes, K. Liu, and T. H. Nieh, Appl. Phys. Lett. 86, 101915 (2005).

${ }^{3}$ M. W. Chen, E. Ma, K. J. Hemker, H. W. Sheng, Y. M. Wang, and X. Cheng, Science 300, 1275 (2003).

${ }^{4}$ X. Z. Liao, F. Zhou, E. J. Lavernia, D. W. He, and Y. T. Zhu, Appl. Phys. Lett. 83, 632 (2003).

${ }^{5}$ H. Rosner, J. Markmann, and J. Weissmuller, Philos. Mag. Lett. 84, 321 (2004).

${ }^{6}$ X. L. Wu, Y. T. Zhu, M. W. Chen, and E. Ma, Scr. Mater. 54, 1685 (2006).
${ }^{7}$ X. L. Wu and E. Ma, Appl. Phys. Lett. 88, 061905 (2006).

${ }^{8}$ X. L. Wu and E. Ma, Appl. Phys. Lett. 88, 231911 (2006).

${ }^{9}$ X. L. Wu and E. Ma, J. Mater. Res. 22, 2241 (2007).

${ }^{10}$ E. Ma, Y. M. Wang, Q. H. Lu, M. L. Sui, L. Lu, and K. Lu, Appl. Phys. Lett. 85, 4932 (2004)

${ }^{11}$ V. Yamakov, D. Wolf, S. R. Phillpot, A. K. Mukherjee, and H. Gleiter, Nature Mater. 3, 43 (2004).

${ }^{12}$ H. Van Swygenhoven, P. M. Derlet, and A. G. Frøseth, Nature Mater. 3, 399 (2004).

${ }^{13}$ V. Yamakov, D. Wolf, S. R. Phillpot, and H. Gleiter, Acta Mater. 50, 5005 (2002).

${ }^{14}$ X. Y. Zhang, X. L. Wu, B. Y. Xia, M. Z. Zhou, S. J. Zhou, and C. Jia, Chin. Phys. Lett. 22, 2335 (2005).

${ }^{15}$ A. J. Cao, Y. G. Wei, and S. X. Mao, Appl. Phys. Lett. 90, 151909 (2007). 